\title{
Infiltration of diametrically polarized macrophages predicts overall survival of patients with gastric cancer after surgical resection
}

\author{
Heng Zhang $\cdot$ Xuefei Wang $\cdot$ Zhenbin Shen $\cdot$ \\ Jiejie Xu · Jing Qin · Yihong Sun
}

Received: 12 March 2014/ Accepted: 23 August 2014/Published online: 18 September 2014

(c) The International Gastric Cancer Association and The Japanese Gastric Cancer Association 2014

\begin{abstract}
Background Tumor-associated macrophages (TAMs), the most predominant tumor-infiltrating immune cells, are emerging prognostic factors and therapeutic targets for personalized therapy against malignant neoplasms. We aimed to evaluate the prognostic significance of diametrically polarized TAMs in gastric cancer and generate a predictive nomogram to refine a risk stratification system. Methods We evaluated polarized functional status of infiltrated TAMs by immunohistochemical staining of CD68, CD11c, and CD206 in 180 consecutive gastric cancer patients from Zhongshan Hospital, Shanghai, China. Prognostic values were assessed in these patients. We created a predictive nomogram by integrating polarized TAMs with the TNM staging system for overall survival of gastric cancer patients.
\end{abstract}

H. Zhang, X. Wang, and Z. Shen contributed equally to this work.

H. Zhang · X. Wang · Z. Shen · J. Qin $(\varangle) \cdot$ Y. Sun $(\bowtie)$ Department of General Surgery, Zhongshan Hospital, Shanghai Medical College of Fudan University, 180 Feng Lin Road, Shanghai 200032, China

e-mail: qin.jing@zs-hospital.sh.cn

Y. Sun

e-mail: sun.yihong@zs-hospital.sh.cn

J. Xu

Key Laboratory of Glycoconjugate Research, Ministry of Health, Shanghai, China

J. Xu $(\bowtie)$

Department of Biochemistry and Molecular Biology, School of Basic Medical Sciences, Shanghai Medical College of Fudan University, 138 Yi Xue Yuan Road, PO Box 103, Shanghai 200032, China

e-mail: jjxufdu@fudan.edu.cn
Results $\mathrm{CD}^{+} 8^{+}$TAMs display polarized programs comprising $\mathrm{CD}_{11 \mathrm{c}^{+}}$proinflammatory macrophages (M1) and $\mathrm{CD}_{206^{+}}$immunosuppressive macrophages (M2) that configure versatile infiltration files in gastric cancer. $\mathrm{CD} 11 \mathrm{c}^{+}$ TAMs negatively correlated with lymph node metastasis $(p=0.012)$, whereas $\mathrm{CD}^{2} 06^{+}$TAMs correlated with the Lauren classification $(p=0.031)$. No prognostic difference was observed for overall survival for CD68 density (high $v s$ low, $p=0.1031$ ), whereas high versus low CD11c density $(p<0.0001)$ and low vs high CD206 density $(p=0.0105)$ indicate better overall survival. Multivariate Cox regression analysis identified CD11c and CD206 as independent prognostic factors $(p<0.001$ and $p=0.030$, respectively), which could be integrated with the TNM staging system to generate a predictive nomogram for patient outcomes.

Conclusion Infiltration of polarized TAMs, a novel identified independent prognostic factor, could be combined with the TNM stage to refine a risk stratification system and better stratify patients with different prognosis. Tipping TAMs to an antitumoral phenotype might be a promising therapeutic target for postoperative treatment.

Keywords Gastric cancer - Tumor-associated macrophages $\cdot$ Prognosis $\cdot$ Nomogram $\cdot$ Overall survival

\section{Introduction}

Although the incidence of gastric cancer has declined for decades, it remains one of the commonest malignant cancers worldwide, and gastric cancer is the second-leading cause of cancer-associated death in both sexes worldwide [1]. Traditionally, the prediction of gastric cancer outcomes is based on tumor-cell-based risk stratification systems, 
such as the TNM staging system, composed of tumor invasion depth, lymph node metastasis, and distant metastasis. However, these clinicopathological factors cannot provide complete prognostic information because they do not incorporate tumor-microenvironment information. Some patients with advanced-stage cancer can remain stable for years, whereas some early-stage patients progress rapidly [2]. Therefore, reliable biomarkers or stratification systems that can be used for more accurate prediction are urgently needed.

Compelling evidence has emerged in recent years that the tumor microenvironment plays a critical role in tumor progression [3]. Macrophages are one of the major components of the tumor microenvironment, are recruited by chemokines such as macrophage colony stimulating factor (M-CSF) and chemokine $\mathrm{C}-\mathrm{C}$ motif ligand 2, and are produced mainly by tumor cells [4]. Macrophages are versatile cells that can express different functional programs in response to microenvironmental signals [5]. Bacterial stimuli, interferon- $\gamma$, and granulocyte-macrophage colony stimulating factor promote a proinflammatory M1-polarized phenotype, whereas M-CSF, interleukin-4, and interleukin-13 favor the generation of folate receptor $\beta$ positive, interleukin-10-producing, immunosuppressive, M2-polarized macrophages [6]. Macrophages that infiltrate tumor tissues, also termed tumor-associated macrophages (TAMs), can be driven by tumor-derived and T-cell-derived cytokines to acquire such a polarized phenotype, and have a key role in subversion of adaptive immunity and in inflammatory circuits that promote tumor growth and progression [7]. Fully polarized M1 and M2 macrophages are the extremes of a continuum of functional states; in contrast to this binary M1/M2 definition, there are several other distinct populations that share features of both types [8]. Functions of these subpopulations in tumor progression need careful elucidation.

There have recently been extensive studies on the correlation between intratumoral macrophages and prognosis, but the results were heterogeneous because they did not discriminate the M1 and M2 macrophage subsets [9-14]. A handful of studies proved that the alternative activated M2 macrophages are the main infiltration files of TAMs in various tumors [15, 16]. The M2-polarized macrophages promote tumor progression and metastasis by activating circuits that regulate tumor growth, adaptive immunity, stroma formation, and angiogenesis, and can be used as prognostic indicators [7, 17, 18]. But the classically activated M1 cells also participate in the crucial process of tumor progression, and mainly provide an antitumor benefit by activating production of toxic intermediates and reactive oxygen intermediates. Characterization of the polarizedTAM-based immune status could be more useful for understanding the tumor-derived signals that guide the polarization of macrophages, and could thus provide a molecular stratification strategy for predicting a more precise prognosis or even predicting the response of patients to specific therapies. According to this knowledge, we hypothesized that polarized-TAM-based immune status may correlate with the progression and prognosis of gastric cancer. Incorporating the immune status into a tumor-cellcentered stratification system may provide a more comprehensive understanding of tumor biology and more precise prognostic significance.

Prognostic nomograms are tools designed for prediction by combining all proven prognostic markers and quantify risk as precisely as possible instead of producing risk groups [19]. In this study, we investigated the prognostic significance of polarized TAMs in gastric cancer and studied its relation with clinical outcomes. On the basis of infiltration of polarized TAMs and the tumor-cell-centered TNM stratification system, we generated a powerful nomogram with high prognostic value in patients with gastric cancer. The results suggested the prognostic significance of macrophage infiltration in gastric cancer, and tipping the balance of polarized TAMs toward an antitumoral phenotype might be a promising target of postoperative adjuvant therapy for patients with gastric cancer.

\section{Methods}

\section{Patients and specimens}

One hundred eighty consecutive gastric cancer patients who received standard gastrectomy with lymph node resection from May 2002 to April 2006 were enrolled in our research with informed consent approved by the Clinical Research Ethics Committee of Zhongshan Hospital of Fudan University. None of these patients received any preoperative anticancer treatment. Baseline clinicopathological features of these patients including age, gender, tumor location, tumor size, tumor differentiation, Lauren classification, and TNM staging were collected retrospectively. Tumor stage and tumor differentiation grade were reclassified according to the seventh American Joint Committee on Cancer TNM classification by two independent gastroenterology pathologists. The clinicopathological features of the 180 gastric cancer patients are shown in Table 1 . The median age of this cohort was 63 years (range 32-83 years), and $68.9 \%$ of the cohort were male; $66.1 \%$ had intestinal-type carcinoma, and the rest had diffuse-type carcinoma; $68.9 \%$ of patients had lymph node metastasis, and six patients $(3.3 \%)$ had resectable distant metastases. Patients were observed until July 2013, with a median observation time of 59 months. Overall survival (OS) was defined as the interval between the dates of surgery and death or the last visit. 
Table 1 Relations between CD68, CD11c, or CD206 expression and clinicopathological characteristics in patients with gastric cancer $(n=180)$

\begin{tabular}{|c|c|c|c|c|c|c|c|c|c|c|c|}
\hline \multirow[t]{2}{*}{ Factors } & \multicolumn{2}{|c|}{ Patients } & \multicolumn{3}{|c|}{ CD68 expression } & \multicolumn{3}{|c|}{ CD11c expression } & \multicolumn{3}{|c|}{ CD206 expression } \\
\hline & No. & $\%$ & $\begin{array}{l}\text { Low } \\
(n=90)\end{array}$ & $\begin{array}{l}\text { High } \\
(n=90)\end{array}$ & $p$ & $\begin{array}{l}\text { Low } \\
(n=90)\end{array}$ & $\begin{array}{l}\text { High } \\
(n=90)\end{array}$ & $p$ & $\begin{array}{l}\text { Low } \\
(n=91)\end{array}$ & $\begin{array}{l}\text { High } \\
(n=89)\end{array}$ & $p$ \\
\hline Mean age (years) & & & 62.6 & 61.6 & 0.553 & 63.4 & 60.8 & 0.102 & 62.5 & 61.7 & 0.620 \\
\hline Gender & & & & & 0.107 & & & 0.334 & & & 0.087 \\
\hline Female & 56 & 31.1 & 23 & 33 & & 25 & 31 & & 23 & 33 & \\
\hline Male & 124 & 68.9 & 67 & 57 & & 65 & 59 & & 68 & 56 & \\
\hline Localization & & & & & 0.245 & & & 0.402 & & & 0.570 \\
\hline Proximal & 30 & 16.7 & 15 & 15 & & 12 & 18 & & 16 & 14 & \\
\hline Middle & 58 & 32.2 & 34 & 24 & & 32 & 26 & & 32 & 26 & \\
\hline Distal & 92 & 51.1 & 41 & 51 & & 46 & 46 & & 43 & 49 & \\
\hline Tumor size $^{\mathrm{a}}$ & & & & & 0.881 & & & 0.655 & & & 0.762 \\
\hline$<4 \mathrm{~cm}$ & 93 & 51.7 & 47 & 46 & & 48 & 45 & & 46 & 47 & \\
\hline$\geq 4 \mathrm{~cm}$ & 87 & 48.3 & 43 & 44 & & 42 & 45 & & 45 & 42 & \\
\hline Differentiation & & & & & 0.065 & & & 0.682 & & & 0.083 \\
\hline Good & 6 & 3.3 & 5 & 1 & & 2 & 4 & & 5 & 1 & \\
\hline Moderate & 67 & 37.2 & 38 & 29 & & 33 & 34 & & 38 & 29 & \\
\hline Poor & 107 & 59.5 & 47 & 60 & & 55 & 52 & & 48 & 59 & \\
\hline Lauren classification & & & & & 0.637 & & & 0.270 & & & 0.031 \\
\hline Intestinal type & 119 & 66.1 & 61 & 58 & & 56 & 63 & & 67 & 52 & \\
\hline Diffuse type & 61 & 33.9 & 29 & 32 & & 34 & 27 & & 24 & 37 & \\
\hline T classification & & & & & 0.067 & & & 0.295 & & & 0.434 \\
\hline $\mathrm{T} 1$ & 32 & 17.8 & 18 & 14 & & 13 & 19 & & 17 & 15 & \\
\hline $\mathrm{T} 2$ & 15 & 8.3 & 9 & 6 & & 5 & 10 & & 8 & 7 & \\
\hline $\mathrm{T} 3$ & 11 & 6.1 & 9 & 2 & & 6 & 5 & & 8 & 3 & \\
\hline $\mathrm{T} 4$ & 122 & 67.8 & 54 & 68 & & 66 & 56 & & 58 & 64 & \\
\hline $\mathrm{N}$ classification & & & & & 0.058 & & & 0.012 & & & 0.326 \\
\hline N0 & 56 & 31.1 & 35 & 21 & & 24 & 32 & & 33 & 23 & \\
\hline N1 & 43 & 23.9 & 18 & 25 & & 15 & 28 & & 21 & 22 & \\
\hline $\mathrm{N} 2$ & 28 & 15.6 & 16 & 12 & & 19 & 9 & & 15 & 13 & \\
\hline N3 & 53 & 29.4 & 21 & 32 & & 32 & 21 & & 22 & 31 & \\
\hline Distant metastasis & & & & & 0.211 & & & 0.682 & & & 0.116 \\
\hline No & 174 & 96.7 & 89 & 85 & & 86 & 88 & & 90 & 84 & \\
\hline Yes & 6 & 3.3 & 1 & 5 & & 4 & 2 & & 1 & 5 & \\
\hline TNM stage & & & & & 0.102 & & & 0.413 & & & 0.168 \\
\hline I & 38 & 21.1 & 21 & 17 & & 15 & 23 & & 19 & 19 & \\
\hline II & 35 & 19.5 & 22 & 13 & & 17 & 18 & & 22 & 13 & \\
\hline III & 101 & 56.1 & 46 & 55 & & 54 & 47 & & 49 & 52 & \\
\hline IV & 6 & 3.3 & 1 & 5 & & 4 & 2 & & 1 & 5 & \\
\hline
\end{tabular}

$T$ tumor depth, $N$ lymph node, TNM tumor-node-metastasis

a Split at median

Immunohistochemistry and evaluation

CD68 [20], CD11c [21-23], and the mannose receptor CD206 [24], which represented overall infiltrated, M1polarized, and M2-polarized TAMs, respectively, were used to identify and quantify infiltration of polarized TAMs in gastric cancer by immunohistochemical (IHC) staining. The tissue microarray and immunohistochemistry protocols were constructed as described previously [25, 26]. Primary antibodies were against CD68 (Dako, Glostrup, Denmark), CD11c (Abcam, Cambridge, MA, USA), and CD206 (Abcam, Cambridge, MA, USA). The density of positive 
staining was measured with the use of a computerized image system composed of an Olympus CCD camera connected to a Nikon eclipse Ti-s microscope. The IHC staining sections were scanned at high magnification $(\times 200)$ and captured by NIS-Elements F3.2 to identify the five independent microscopic fields with the densest infiltration of TAMs to ensure representativeness and homogeneity. Identical settings were used for each photograph. The density was counted by Image-Pro Plus version 6.0 (Media Cybernetics, Bethesda, MD, USA). The integrated optical density of all the positive staining in each photograph was measured, and its ratio to the total area of each photograph was calculated as the relative density. The results were expressed as the mean density for one computerized microscopic field. The evaluation of immunostaining was performed by two independent gastroenterology pathologists who were blinded to the patient outcomes and clinicopathological characteristics. Variations in the enumeration, within a range of $5 \%$, were reevaluated, and a consensus decision was made. For IHC density, the cutoff for the definition of high/low expression subgroups was the median value.

\section{Statistical analysis}

Analysis was performed with SPSS 19.0 (SPSS, Chicago, IL, USA) and R (http://www.r-project.org/). Pearson's $\chi^{2}$ test or Fisher's exact test was used to compare qualitative variables, and quantitative variables were analyzed by the $t$ test or Pearson's correlation test. Kaplan-Meier analysis was used to determine the survival. The log-rank test was used to compare patient survival between subgroups. The stepwise Cox regression model was used to perform multivariate analysis. Numbers at risk were calculated for the beginning of each time period. A nomogram was created with $\mathrm{R}$ using the "rms" package. A calibration plot was generated to examine the performance characteristics of the nomogram. The bootstrap-corrected concordance index and the Akaike information criterion were used to compare the prognostic accuracy. All statistical analyses were two-sided, and $p<0.05$ was considered statistically significant. Results are reported according to Reporting Recommendations for Tumor Marker Prognostic Studies (REMARK) guidelines [27].

\section{Results}

\section{IHC findings}

The positive staining of CD68, CD11c, and CD206 seen in the cytoplasm and/or membrane of macrophages and confined to the accessory cells surrounding the tumor cells in a diffuse manner confirmed the specificity of these markers
Fig. 1 Versatile polarization status of intratumoral tumor-associated macrophages (TAMs) in gastric cancer patients. Consecutive sections were used for immunohistochemical study: a CD68 high, CD11c low, CD206 high; b CD68 high, CD11c high, CD206 low; c CD68 high, CD11c high, CD206 high; d CD68 low, CD11c low, CD206 low. The scatter plots for immunohistochemistry scores of CD68, CD11c and CD206 in different TNM stages are also shown (e). Positive macrophages were stained brown $(\times 200$ magnification $)$

in indicating TAMs (Fig. 1, panels a-d). CD11 ${ }^{+}$and $\mathrm{CD}_{206}{ }^{+}$macrophages existing within the area where $\mathrm{CD} 8^{+}$macrophages were located confirmed that the $\mathrm{CD} 11 \mathrm{c}^{+}$and $\mathrm{CD} 206^{+}$cells are subsets of $\mathrm{CD}^{+} 8^{+}$macrophages (Fig. 1, panels a-d). We evaluated the density of positive staining in sequential slides per specimen, and found that the infiltration files of polarized TAMs varied largely. The relative density of IHC staining was $2.03 \pm 2.11$ (median, 1.45; range, 0.15-21.4), $0.44 \pm 0.79$ (median, 0.21; range, 0-7.15), and $1.58 \pm 1.93$ (median, 1.10; range, 0.12-20.9) for CD68, CD11c, and CD206, respectively. Some patients had a higher M2 infiltration (Fig. 1, panel a), some had a higher M1 infiltration (Fig. 1, panel b), and some had a high infiltration or a low infiltration of both M1 and M2 (Fig. 1, panels c and d, respectively). The IHC density of CD68, CD11c, and CD206, exhibits no significant difference among samples of different TNM stage (Fig. 1, panel e).

Correlations between TAM polarization status and clinicopathological features

Correlations between IHC variables and clinicopathological features are summarized in Table 1. CD11c staining was negatively correlated with the involvement of lymph nodes $(p=0.012)$. CD206 staining was correlated with the Lauren classification $(p=0.031)$. No association between IHC variables and other clinicopathological factors was observed.

Prognostic value of TAM polarization status

Using median values obtained from the IHC density as a cutoff, we performed Kaplan-Meier analysis. As shown in Fig. 2, low CD11c expression or high CD206 expression was associated with reduced survival (Fig. 2b, c; $p<0.0001$ and $p=0.0105$, respectively), whereas CD68 staining in tumor has no significant relation with OS (Fig. 2a; $p=0.1031$ ). To investigate further the effect of polarized TAMs in stratifying patients with different TNM stages, we considered TNM stages I and II as early-stage tumor, and TNM stages III and IV as advanced-stage tumor. CD11c was positively correlated with OS and CD206 had no significant relation with OS in patients with early-stage tumor (Fig. 3a, b; $p=0.0187$ and $p=0.6093$, 
a

b

C

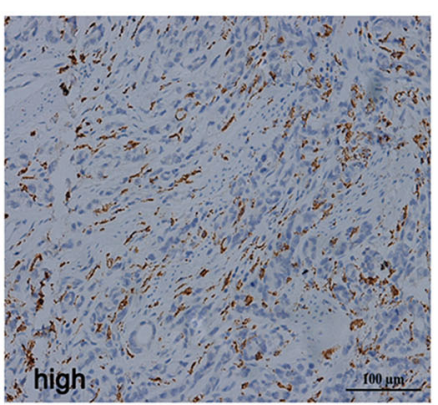

b

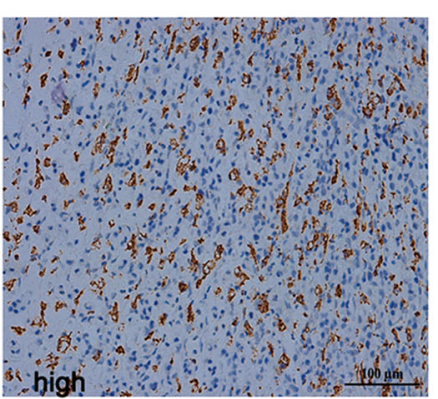

d


e

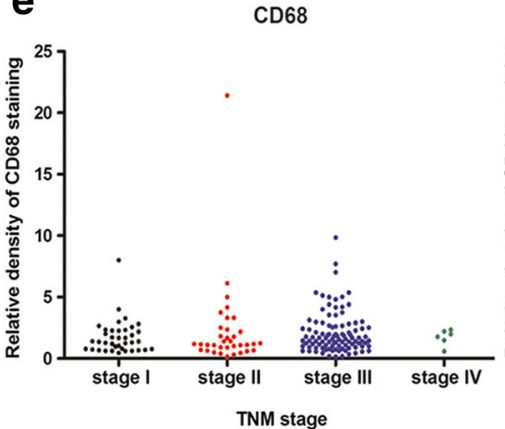

CD11c
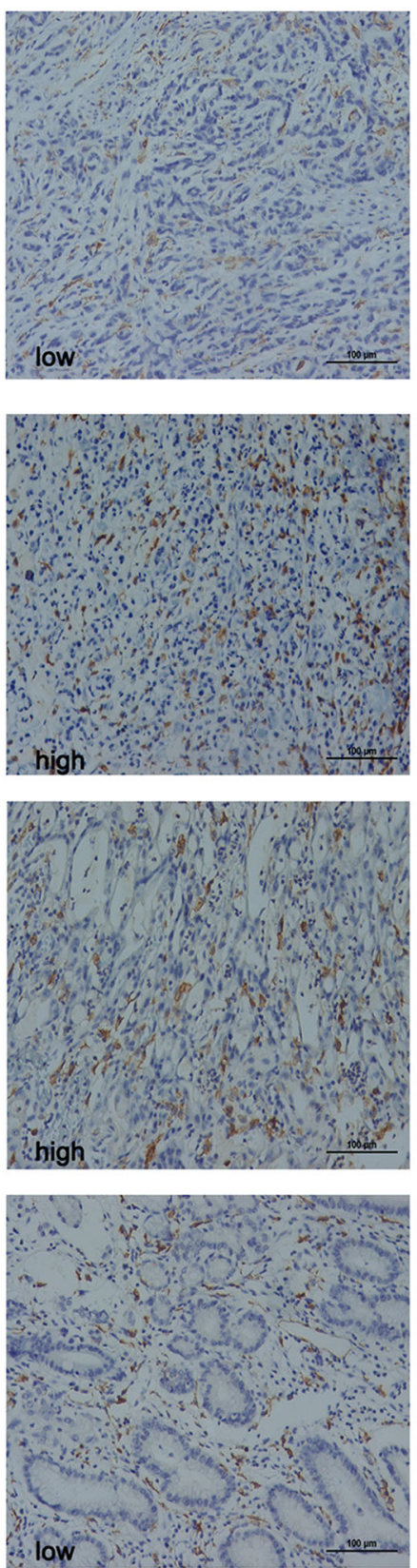

CD11c

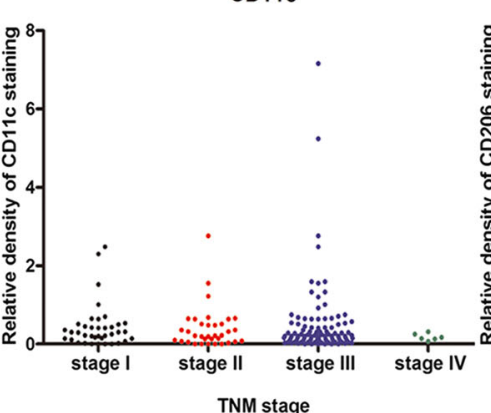

CD206
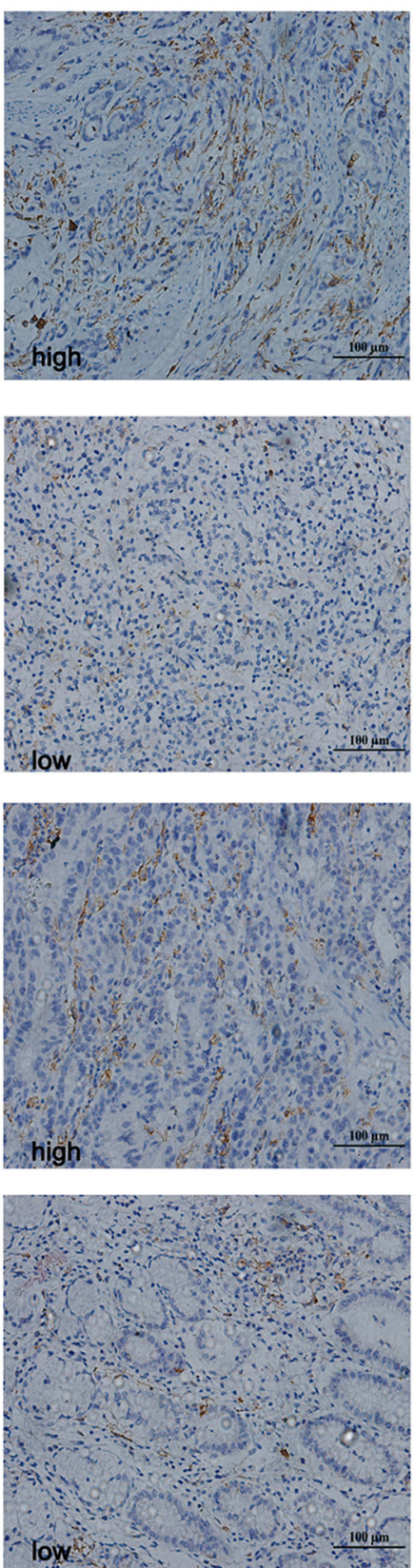

CD206

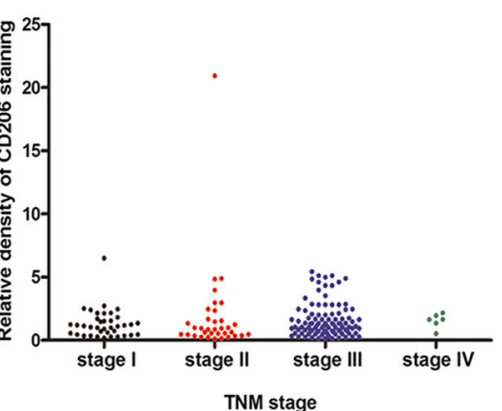


respectively). Moreover, CD11c was positively correlated with OS and CD206 was negatively correlated with OS in patients with advanced-stage tumor (Fig. $3 \mathrm{c}, \mathrm{d} ; p=0.0007$ and $p=0.0126$, respectively). These results suggested that polarized TAMs might provide additional prognostic information in different tumor stages.

\section{Univariate and multivariate survival analysis}

The clinical factors statistically significantly associated with reduced OS (Table 2) were tumor size $(p=0.002)$, T classification $(p<0.001), \mathrm{N}$ classification $(p<0.001)$, distant metastasis $(p<0.001)$, TNM stage $(p<0.001)$, CD11c expression $(p<0.001)$, and CD206 expression $(p=0.012)$. Variables demonstrating a significant effect on OS were included in the multivariate analysis. Moreover, to avoid confounding factors, TNM stage was excluded in the multivariate analysis. $\mathrm{T}$ classification $(p=0.010)$, $\mathrm{N}$ classification $(p=0.008)$, distant metastasis $(p=0.007), \mathrm{CD} 11 \mathrm{c}$ expression $(p<0.001)$, and CD206 expression $(p=0.030)$ were identified as independent prognostic factors for OS after adjustment of covariates (Table 2).

Predictive nomogram model for overall survival of gastric cancer patients

To provide a quantitative method to better stratify patients with different prognosis, we constructed a nomogram that integrated the proven prognostic factor consisting of tumor invasion depth, lymph node involvement, distant metastasis, and polarized macrophage infiltration (Fig. 4a). In this nomogram, a higher total number of points indicates poorer prognosis. The calibration plot showed that the nomogram performs well compared with the ideal prediction model (Fig. 4b). The bootstrap-corrected concordance index for OS was 0.7426 in the nomogram model compared with 0.6872 in the TNM stage model, and the Akaike information criterion was reduced to 816.7575 compared with 832.4341 in the TNM model. A wide range of predicted survival could be identified in each TNM stage (Fig. 4c). Furthermore, the range of predicted survival was wider for earlier stages. All these results indicated that incorporation of polarized TAMs into the TNM staging system could further stratify patients with different prognosis.

\section{Discussion}

This study proved the hypothesis that the infiltration of diametrically polarized TAMs influences OS of patients with surgically resected gastric cancer. In this study, we

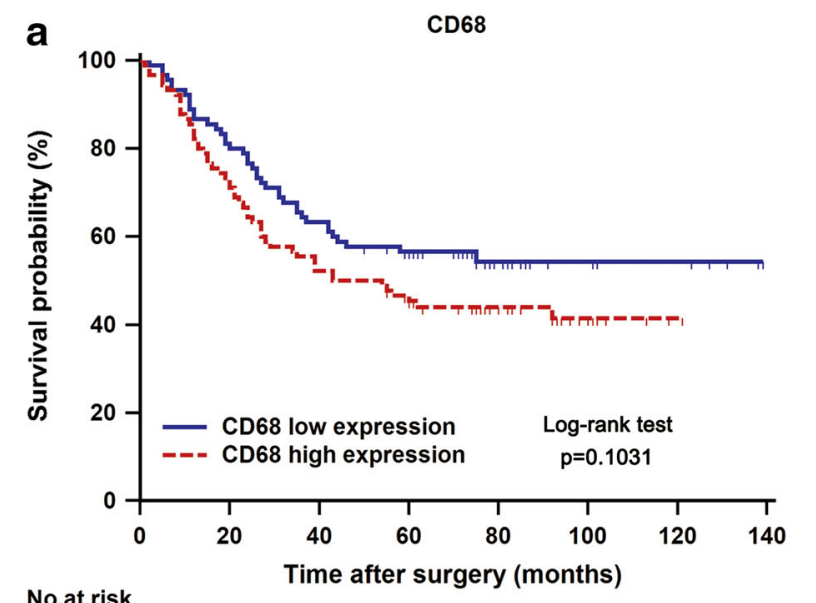

No.at risk

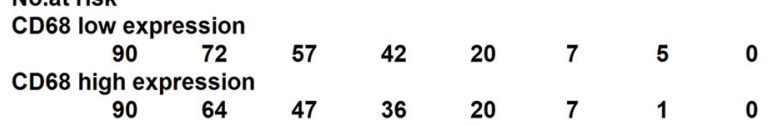

b CD11c

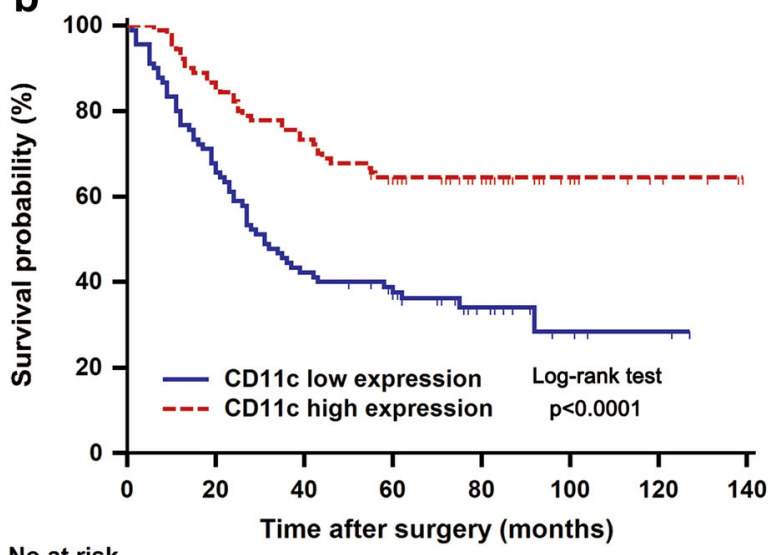

No.at risk

CD11c low expression

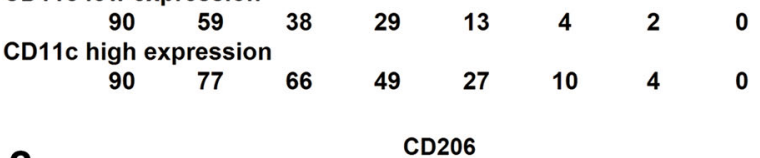

C

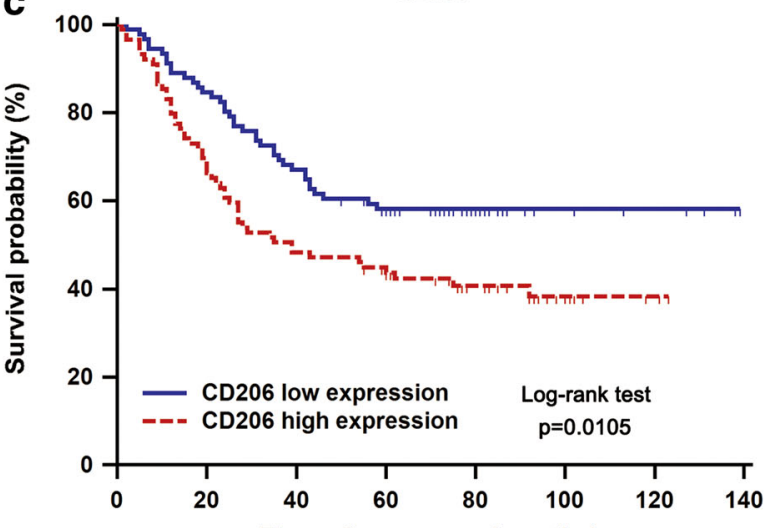

No.at risk

Time after surgery (months)

CD206 low expression

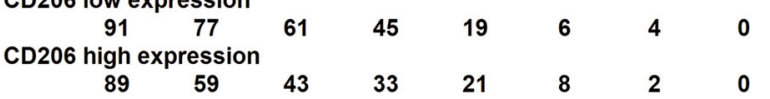


4Fig. 2 Kaplan-Meier survival curves for gastric cancer patients with diametrically polarized TAMs: a CD68 (high $v s$ low, $p=0.1031$ ); b CD11c (high vs low, $p<0.0001$ ); c CD206 (high vs low, $p=0.0105)$. The $p$ values were calculated by the log-rank test

observed that TAMs indicated by CD68 positive staining irrespective of functional status have no significant correlation with OS, whereas single use of CD11c or CD206 has a significant positive and negative correlation with OS, respectively. Cox regression analysis confirmed that the polarized TAMs emerged as an independent prognostic factor. Incorporating the polarized TAM system into the established TNM system generates a nomogram that can quantify the prognostic risk more precisely. However, the incorporation of macrophage infiltration into current prognostic models requires independent and more data to validate it.

There is growing appreciation that inflammation in the tumor microenvironment is the root of many malignancies [28]. Activated macrophages are central to tumor-associated inflammation in combination with other immune cells, which has been considered as proof that the host interacts with the growing tumor [29]. But the precise infiltration files of these immune cells and their functional status are still obscure. In this study, we focused on TAMs, and found that the diametrically polarized functional status of

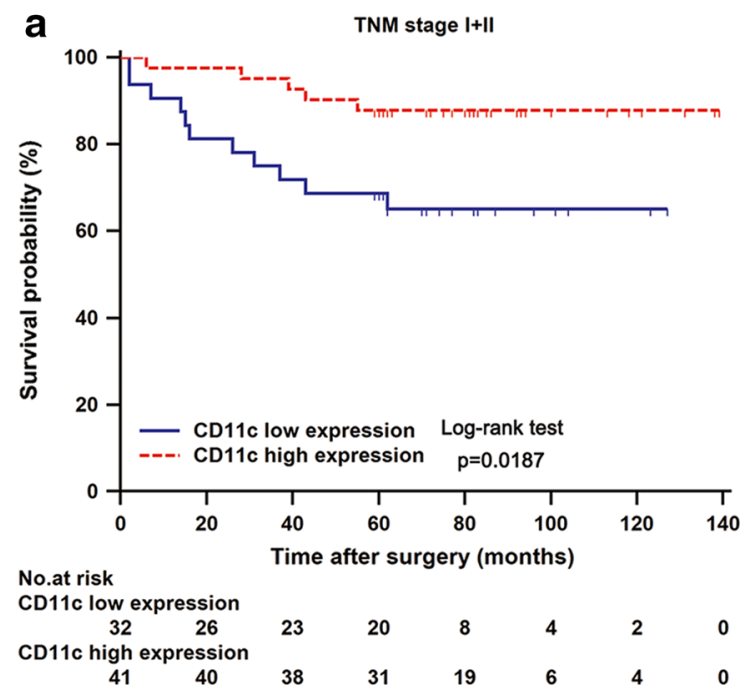

b

b TNM stage I+II
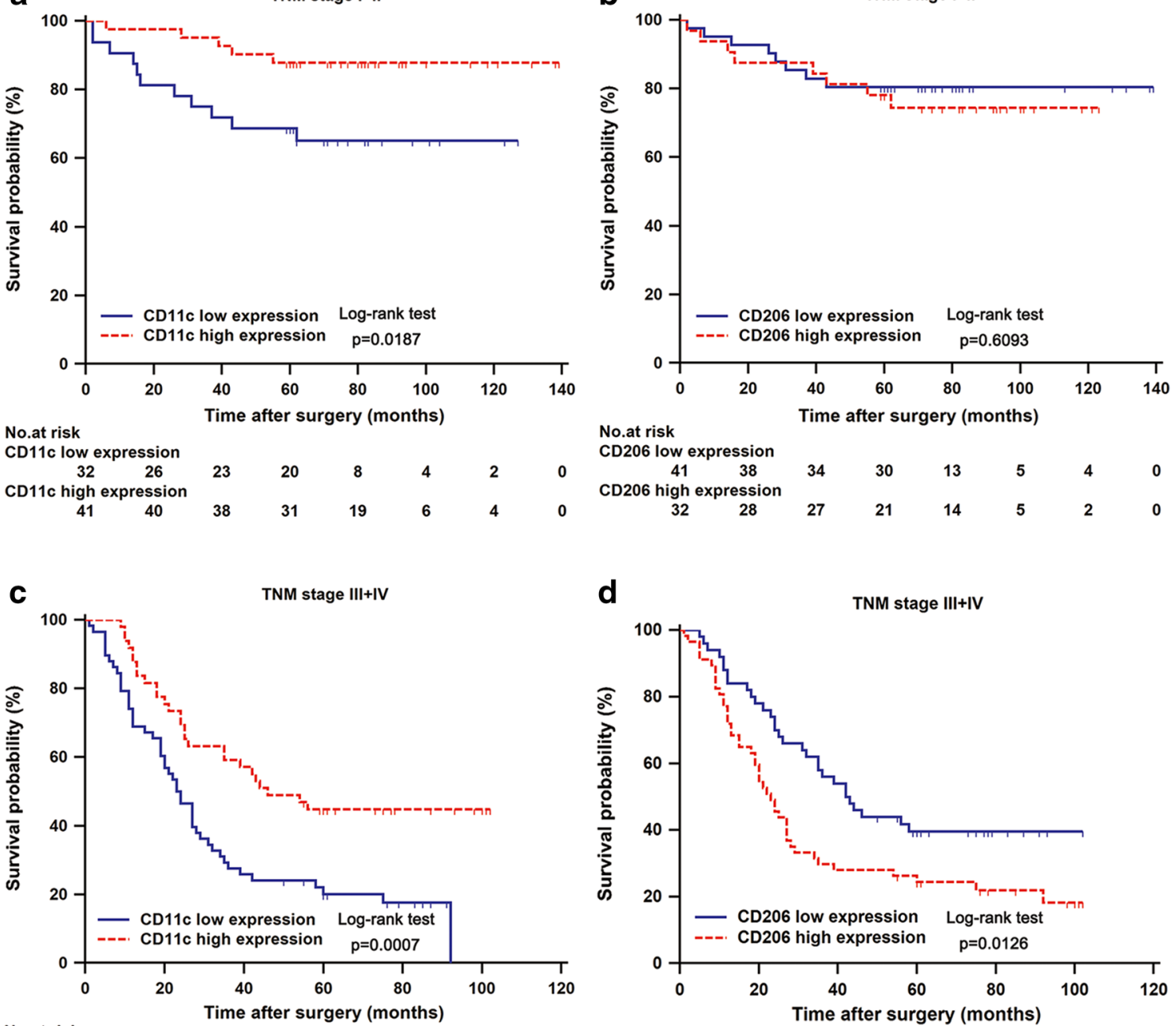

No.at risk

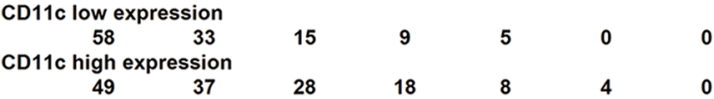

d

TNM stage III+IV

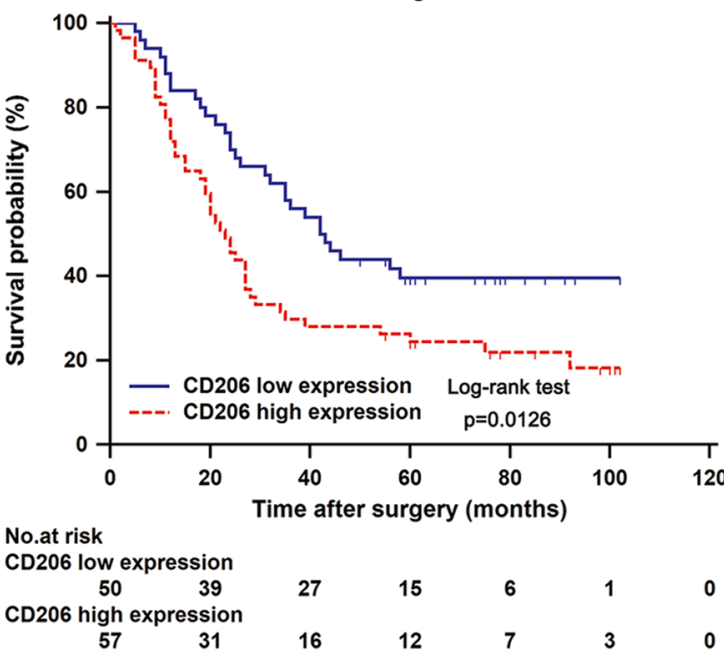

expression (high $v s$ low, $p=0.6093$ ); $\mathbf{c}$ patients with advanced-stage tumor (TNM stages III and IV) according to CD11c expression (high vs low, $p=0.0007$ ); d patients with advanced-stage tumor (TNM stages III and IV) according to CD206 expression (high vs low, $p=0.0126)$. The $p$ values were calculated by the log-rank test 
Table 2 Univariate and multivariate Cox regression analyses for overall survival of patients with gastric cancer $(n=180)$

\begin{tabular}{|c|c|c|c|c|}
\hline \multirow[t]{2}{*}{ Factors } & \multicolumn{2}{|l|}{ Univariate } & \multicolumn{2}{|l|}{ Multivariate } \\
\hline & Hazard ratio $(95 \% \mathrm{CI})$ & $p$ & Hazard ratio $(95 \% \mathrm{CI})$ & $p$ \\
\hline Age & $1.014(0.995-1.034)$ & 0.154 & & \\
\hline Gender & & 0.570 & & \\
\hline Female & 1 (reference) & & & \\
\hline Male & $0.878(0.559-1.377)$ & & & \\
\hline Localization & & 0.127 & & \\
\hline Middle vs proximal & $0.594(0.320-1.101)$ & 0.098 & & \\
\hline Distal vs proximal & $0.957(0.558-1.642)$ & 0.875 & & \\
\hline Differentiation & & 0.308 & & \\
\hline Moderate vs good & $3.382(0.463-24.731)$ & 0.230 & & \\
\hline Poor vs good & $4.015(0.555-29.035)$ & 0.169 & & \\
\hline Lauren classification & & 0.208 & & \\
\hline Intestinal & 1 (reference) & & & \\
\hline Diffuse & $1.314(0.859-2.010)$ & & & \\
\hline Tumor size $^{a}$ & & 0.002 & & 0.096 \\
\hline$<4 \mathrm{~cm}$ & 1 (reference) & & 1 (reference) & \\
\hline$\geq 4 \mathrm{~cm}$ & $1.969(1.290-3.006)$ & & $1.454(0.936-2.260)$ & \\
\hline $\mathrm{T}$ classification & & $<0.001$ & & 0.010 \\
\hline $\mathrm{T} 1$ & 1 (reference) & & 1 (reference) & \\
\hline $\mathrm{T} 2+\mathrm{T} 3+\mathrm{T} 4$ & $14.478(3.559-58.889)$ & & $6.863(1.585-29.712)$ & \\
\hline $\mathrm{N}$ classification & & $<0.001$ & & 0.008 \\
\hline No & 1 (reference) & & 1 (reference) & \\
\hline $\mathrm{N} 1+\mathrm{N} 2+\mathrm{N} 3$ & $5.368(2.775-10.383)$ & & $2.570(1.286-5.135)$ & \\
\hline Distant metastasis & & $<0.001$ & & 0.007 \\
\hline No & 1 (reference) & & 1 (reference) & \\
\hline Yes & $5.746(2.424-13.617)$ & & $3.353(1.401-8.023)$ & \\
\hline TNM stage & & $<0.001$ & & \\
\hline II vs I & $3.939(1.268-12.236)$ & 0.018 & & \\
\hline III vs I & $10.504(3.820-28.885)$ & $<0.001$ & & \\
\hline IV vs I & $39.645(10.806-145.452)$ & $<0.001$ & & \\
\hline CD68 expression & & 0.107 & & \\
\hline Low & 1 (reference) & & & \\
\hline High & $1.407(0.929-2.129)$ & & & \\
\hline CD11c expression & & $<0.001$ & & $<0.001$ \\
\hline Low & 1 (reference) & & 1 (reference) & \\
\hline High & $0.399(0.259-0.615)$ & & $0.422(0.272-0.654)$ & \\
\hline CD206 expression & & 0.012 & & 0.030 \\
\hline Low & 1 (reference) & & 1 (reference) & \\
\hline High & $1.709(1.125-2.595)$ & & $1.610(1.047-2.477)$ & \\
\hline
\end{tabular}

macrophages configured a complex immune status in predicting prognosis of gastric cancer patients.

Macrophages derived from the $\mathrm{CD} 34^{+}$bone marrow progenitors develop into monocytes, and extravasate into various tissues, where they differentiate into resident macrophages [15]. The monocyte-macrophage lineage is diverse and plastic, attributed to a complex transcriptome [30]. To facilitate immune function and maintain tissue homeostasis, macrophages adopt a wide range of activation states that can be classified within the classic activated M1 and alternative activated M2 models of macrophage polarization by interaction with the tumor-microenvironment milieu [31]. Accumulating evidence indicates that TAMs play an important role in tumor initiation, progression, and metastasis [32]. However, the polarized TAMs have diametrically different functions in these processes. 


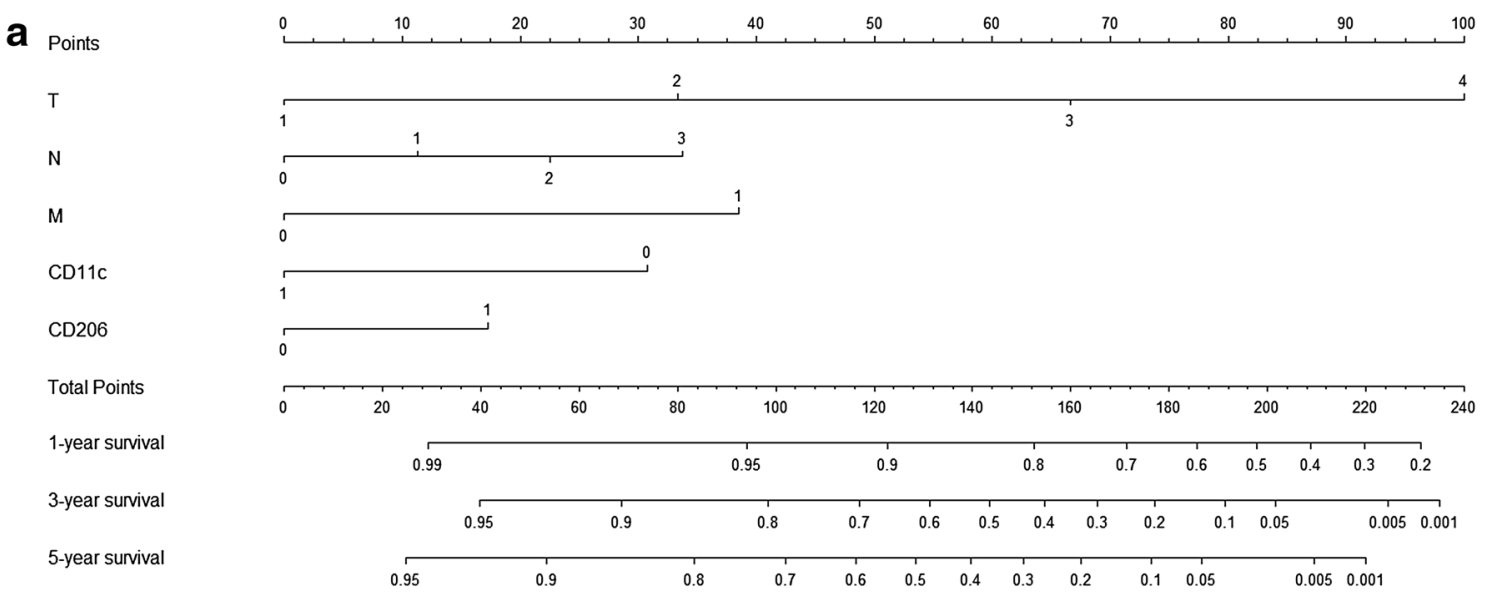

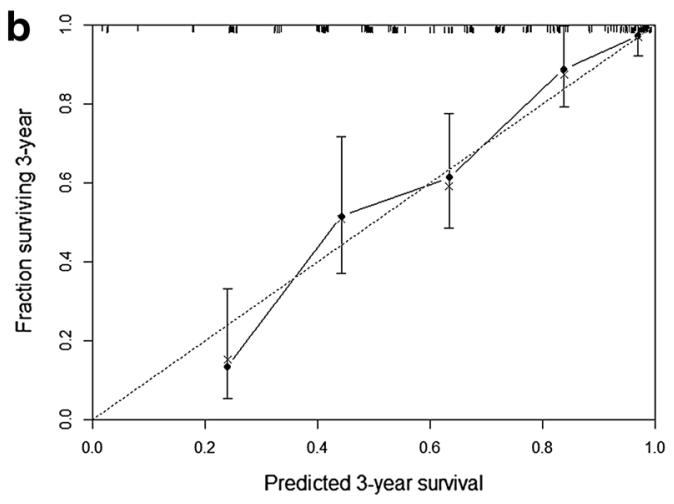

Fig. 4 Prognostic nomogram to predict overall survival in patients with gastric cancer. a Nomogram generation for predicting outcomes integrated with polarized TAMs (CD11c and CD206; 0 represents low expression, 1 represents high expression), tumor invasion depth ( $T$; 1 represents $\mathrm{T} 1,2$ represents $\mathrm{T} 2,3$ represents $\mathrm{T} 3,4$ represents T4), lymph node involvement $(N$; 0 represents N0, 1 represents $\mathrm{N} 1$, 2 represents $\mathrm{N} 2,3$ represents $\mathrm{N} 3)$, and distant metastasis $(M$;

The M1-skewed macrophages are able to release large amounts of proinflammatory cytokines, reactive nitrogen intermediates, and reactive oxygen intermediates, have higher expression of major histocompatibility complex class II and costimulatory molecules, and have efficient antigen presentation to favor their tumoricidal activity, whereas the M2-skewed macrophages produce molecules that promote tumor cell growth directly, enhance angiogenesis, tune inflammatory responses and adaptive immunity, and catalyze structural and substantial changes of the extracellular matrix compartment to facilitate tumor metastasis [33]. The functional status of TAMs in tumor is not immutable, and there is a dynamic switch. The local cytokine milieu in the tumor microenvironment is important to the phenotype switch of those infiltrated TAMs. With tumor progression, cytokines derived from tumor cells and their surrounding microenvironment, such as

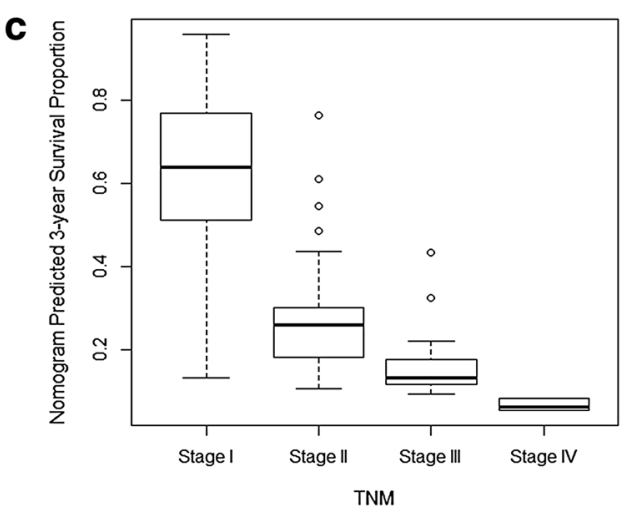

0 represents absence, 1 represents presence). b Calibration curve for nomogram-predicted and observed 3-year outcomes. c The box plot represents the distribution of nomogram-predicted 3-year survival according to seventh American Joint Committee on Cancer TNM classification. A wide range of predicted survival can be identified in each TNM stage

M-CSF, educate macrophages to perform trophic functions and adopt an immunosuppressive M2-skewed macrophage phenotype [32, 34]. Moreover, variations on the typical M1/M2 phenotype have also been noted depending on the tumor type and organ involved. Once resident in tissues, macrophages acquire a distinct, tissue-specific phenotype in response to different microenvironment signals present within individual tumors [35-37].Thus, the infiltration files and prognostic significance of TAMs in gastric cancer may differ from those of other tumors and need further investigation.

Studies attempting to elucidate the prognostic significance of TAMs have generated heterogeneous results, most likely owing to not discriminating the M1/M2 subsets [38]. Many studies have identified the important role of TAMs in gastric cancer. Wu et al. [39] found that TAMs could promote angiogenesis and lymphangiogenesis of gastric 
cancer via elevated levels of vascular endothelial growth factor and vascular endothelial growth factor C. Okita et al. [40] proved the prognostic significance of antigen-presenting cells in patients with gastric cancer. Ishigami et al. [41] found a negative correlation between TAMs and prognosis in gastric cancer, and Ohno et al. [14, 41] revealed that the aggregation of TAMs within the tumor nest had a beneficial effect. These studies have proved the prognostic significance of TAMs in gastric cancer; however, the diametrically opposed functional status of TAMs was beyond these investigations. A recent study in renal cell carcinoma tried to prove the prognostic significance of M1/M2 phenotypes using combined analysis of CD11c and CD206 [42]. This may be better to help elucidate the prognostic significance of diametrically polarized infiltrated macrophages. In the present study, we investigated the prognostic significance of the M1/M2 phenotype in patients with gastric cancer and found that the M1/M2 phenotype may better stratify patients with different prognosis compared with overall infiltrated TAMs. Moreover, diametrically polarized infiltrated macrophages can be incorporated with TNM staging parameters to generate a predictive nomogram for OS with better accuracy. These results may help clinicians to better stratify patients who need more aggressive adjuvant therapy or who need more intensive follow-up.

In our effort to clarify the infiltration of polarized TAMs in gastric cancer progression, we found that patients with low CD11c expression developed lymph node metastasis more easily, whereas CD206 expression correlated with the Lauren classification (Table 1). These results partially revealed the effects of polarized TAMs mediated the tumor cell biological phenotype. Although there are many biological factors of innate tumor that determine tumor biological behaviors, more and more evidence has suggested that the tumor microenvironment also plays critical roles in determining tumor biology [32, 43]. In this study, we have proved that the infiltration of polarized TAMs (the most abundant immune cells in the microenvironment) in gastric cancer is correlated with lymph node metastasis and can be used to stratify patients with different prognosis, even patients with disease of the same stage. Moreover, adding the prognostic information derived from polarized TAMs to the TNM staging system could provide additional prognostic information that might be used in better distinguishing patient outcomes and making corresponding follow-ups.

Along with the predictive significance of myeloid immune cell infiltration, accumulating evidence indicates that the success of anticancer therapies, including conventional cytotoxic compounds, radiotherapy, and targeted agents, depends (at least partially) on the activation of anticancer immune responses [44]. The phenotype of polarized M1/M2 macrophages can, to some extent, be reversed in vitro and in vivo $[45,46]$. Thus, restoration of the complex immune system to the antitumor state by blocking the transition alone or in combination with chemotherapy may open a new avenue for treatment of patients with gastric cancer.

In conclusion, our study focused on the prognostic significance of polarized-TAM-based immune status in gastric cancer. Combining the polarized TAMs with the proven TNM stage can generate a nomogram that may become a useful prognostic tool in prognosis, follow-up, and intervention counseling for patients with gastric cancer.

Acknowledgments The authors thank Haiying Zeng and Rongkui Luo (Department of Pathology, Zhongshan Hospital) for experimental technical help. This work was supported by grants from the National Key Projects for Infectious Diseases of China (2012ZX10002-012), the National Natural Science Foundation of China (31100629, $31270863,31300671,31470794,81472227,81471621$ ), the Program for New Century Excellent Talents in University (NCET-13-0146), the Shanghai Rising-Star Program (13QA1400300), the Key Project of Science and Technology Commission of Shanghai Municipality (09DZ1950101, 11411951000, 12140902000), and the Research Fund for Excellent Doctor of Shanghai Medical College of Fudan University (EZF152309001003001).

Conflict of interest The authors made no disclosures.

\section{References}

1. Hartgrink HH, Jansen EP, van Grieken NC, van de Velde CJ. Gastric cancer. Lancet. 2009;374:477-90.

2. Fridman WH, Mlecnik B, Bindea G, Pages F, Galon J. Immunosurveillance in human non-viral cancers. Curr Opin Immunol. 2011;23:272-8.

3. Liotta LA, Kohn EC. The microenvironment of the tumour-host interface. Nature. 2001;411:375-9.

4. Wiktor-Jedrzejczak W, Gordon S. Cytokine regulation of the macrophage (M phi) system studied using the colony stimulating factor-1-deficient op/op mouse. Physiol Rev. 1996;76:927-47.

5. Mantovani A, Sica A, Sozzani S, Allavena P, Vecchi A, Locati M. The chemokine system in diverse forms of macrophage activation and polarization. Trends Immunol. 2004;25:677-86.

6. Murray PJ, Wynn TA. Protective and pathogenic functions of macrophage subsets. Nat Rev Immunol. 2011;11:723-37.

7. Mantovani A, Sozzani S, Locati M, Allavena P, Sica A. Macrophage polarization: tumor-associated macrophages as a paradigm for polarized M2 mononuclear phagocytes. Trends Immunol. 2002;23:549-55.

8. Ojalvo LS, King W, Cox D, Pollard JW. High-density gene expression analysis of tumor-associated macrophages from mouse mammary tumors. Am J Pathol. 2009;174:1048-64.

9. Lissbrant IF, Stattin P, Wikstrom P, Damber JE, Egevad L, Bergh A. Tumor associated macrophages in human prostate cancer: relation to clinicopathological variables and survival. Int J Oncol. 2000; $17: 445-51$.

10. Ohno S, Ohno Y, Suzuki N, Kamei T, Koike K, Inagawa H, et al. Correlation of histological localization of tumor-associated macrophages with clinicopathological features in endometrial cancer. Anticancer Res. 2004;24:3335-42. 
11. Hanada T, Nakagawa M, Emoto A, Nomura T, Nasu N, Nomura Y. Prognostic value of tumor-associated macrophage count in human bladder cancer. Int J Urol. 2000;7:263-9.

12. Lee CH, Espinosa I, Vrijaldenhoven S, Subramanian S, Montgomery KD, Zhu S, et al. Prognostic significance of macrophage infiltration in leiomyosarcomas. Clin Cancer Res. 2008;14:1423-30.

13. Forssell J, Oberg A, Henriksson ML, Stenling R, Jung A, Palmqvist R. High macrophage infiltration along the tumor front correlates with improved survival in colon cancer. Clin Cancer Res. 2007;13:1472-9.

14. Ohno S, Inagawa H, Dhar DK, Fujii T, Ueda S, Tachibana M, et al. The degree of macrophage infiltration into the cancer cell nest is a significant predictor of survival in gastric cancer patients. Anticancer Res. 2003;23:5015-22.

15. Kurahara H, Shinchi H, Mataki Y, Maemura K, Noma H, Kubo F, et al. Significance of M2-polarized tumor-associated macrophage in pancreatic cancer. J Surg Res. 2011;167:e211-9.

16. Sica A, Schioppa T, Mantovani A, Allavena P. Tumour-associated macrophages are a distinct M2 polarised population promoting tumour progression: potential targets of anti-cancer therapy. Eur J Cancer. 2006;42:717-27.

17. Balkwill $\mathrm{F}$, Mantovani A. Inflammation and cancer: back to Virchow? Lancet. 2001;357:539-45.

18. Coussens LM, Tinkle CL, Hanahan D, Werb Z. MMP-9 supplied by bone marrow-derived cells contributes to skin carcinogenesis. Cell. 2000;103:481-90.

19. Kattan MW, Karpeh MS, Mazumdar M, Brennan MF. Postoperative nomogram for disease-specific survival after an R0 resection for gastric carcinoma. J Clin Oncol. 2003;21:3647-50.

20. Falini B, Flenghi L, Pileri S, Gambacorta M, Bigerna B, Durkop $\mathrm{H}$, et al. PG-M1: a new monoclonal antibody directed against a fixative-resistant epitope on the macrophage-restricted form of the CD68 molecule. Am J Pathol. 1993;142:1359-72.

21. Morris DL, Singer K, Lumeng CN. Adipose tissue macrophages: phenotypic plasticity and diversity in lean and obese states. Curr Opin Clin Nutr Metab Care. 2011;14:341-6.

22. Ito A, Suganami T, Yamauchi A, Degawa-Yamauchi M, Tanaka M, Kouyama R, et al. Role of CC chemokine receptor 2 in bone marrow cells in the recruitment of macrophages into obese adipose tissue. J Biol Chem. 2008;283:35715-23.

23. Nishimura S, Manabe I, Nagasaki M, Eto K, Yamashita H, Ohsugi $\mathrm{M}$, et al. CD8+ effector $\mathrm{T}$ cells contribute to macrophage recruitment and adipose tissue inflammation in obesity. Nat Med. 2009;15:914-20.

24. De Palma M, Lewis CE. Macrophage regulation of tumor responses to anticancer therapies. Cancer Cell. 2013;23:277-86.

25. Zhu XD, Zhang JB, Zhuang PY, Zhu HG, Zhang W, Xiong YQ, et al. High expression of macrophage colony-stimulating factor in peritumoral liver tissue is associated with poor survival after curative resection of hepatocellular carcinoma. J Clin Oncol. 2008;26:2707-16.

26. He H, Wang C, Shen Z, Fang Y, Wang X, Chen W, et al. Upregulated expression of $\mathrm{C}-\mathrm{X}-\mathrm{C}$ chemokine receptor 4 is an independent prognostic predictor for patients with gastric cancer. PLoS One. 2013;8:e71864.

27. McShane LM, Altman DG, Sauerbrei W, Taube SE, Gion M, Clark GM. REporting recommendations for tumour MARKer prognostic studies (REMARK). Eur J Cancer. 2005;41:1690-6.
28. Mantovani A, Sica A. Macrophages, innate immunity and cancer: balance, tolerance, and diversity. Curr Opin Immunol. 2010;22:231-7.

29. Balkwill F, Charles KA, Mantovani A. Smoldering and polarized inflammation in the initiation and promotion of malignant disease. Cancer Cell. 2005;7:211-7.

30. Suzuki H, Forrest AR, van Nimwegen E, Daub CO, Balwierz PJ, Irvine KM, et al. The transcriptional network that controls growth arrest and differentiation in a human myeloid leukemia cell line. Nat Genet. 2009;41:553-62.

31. Martinez FO, Sica A, Mantovani A, Locati M. Macrophage activation and polarization. Front Biosci. 2008;13:453-61.

32. Pollard JW. Tumour-educated macrophages promote tumour progression and metastasis. Nat Rev Cancer. 2004;4:71-8.

33. Biswas SK, Mantovani A. Macrophage plasticity and interaction with lymphocyte subsets: cancer as a paradigm. Nat Immunol. 2010;11:889-96.

34. Pollard JW. Trophic macrophages in development and disease. Nat Rev Immunol. 2009;9:259-70.

35. Lewis CE, Pollard JW. Distinct role of macrophages in different tumor microenvironments. Cancer Res. 2006;66:605-12.

36. Ruffell B, Affara NI, Coussens LM. Differential macrophage programming in the tumor microenvironment. Trends Immunol. 2012;33:119-26.

37. Movahedi K, Laoui D, Gysemans C, Baeten M, Stange G, Van den Bossche J, et al. Different tumor microenvironments contain functionally distinct subsets of macrophages derived from Ly6C(high) monocytes. Cancer Res. 2010;70:5728-39.

38. Senovilla L, Vacchelli E, Galon J, Adjemian S, Eggermont A, Fridman WH, et al. Trial watch: prognostic and predictive value of the immune infiltrate in cancer. Oncoimmunology. 2012;1: $1323-43$.

39. Wu H, Xu JB, He YL, Peng JJ, Zhang XH, Chen CQ, et al. Tumor-associated macrophages promote angiogenesis and lymphangiogenesis of gastric cancer. J Surg Oncol. 2012;106:462-8.

40. Okita Y, Tanaka H, Ohira M, Muguruma K, Kubo N, Watanabe $\mathrm{M}$, et al. Role of tumor-infiltrating $\mathrm{CD} 11 \mathrm{~b}+$ antigen-presenting cells in the progression of gastric cancer. J Surg Res. 2014;186:192-200.

41. Ishigami $S$, Natsugoe $S$, Tokuda $K$, Nakajo A, Okumura $H$, Matsumoto M, et al. Tumor-associated macrophage (TAM) infiltration in gastric cancer. Anticancer Res. 2003;23:4079-83.

42. Xu L, Zhu Y, Chen L, An H, Zhang W, Wang G, et al. Prognostic value of diametrically polarized tumor-associated macrophages in renal cell carcinoma. Ann Surg Oncol. 2014;21:3142-50.

43. Qian BZ, Pollard JW. Macrophage diversity enhances tumor progression and metastasis. Cell. 2010;141:39-51.

44. Galluzzi L, Senovilla L, Zitvogel L, Kroemer G. The secret ally: immunostimulation by anticancer drugs. Nat Rev Drug Discov. 2012;11:215-33.

45. Saccani A, Schioppa T, Porta C, Biswas SK, Nebuloni M, Vago $\mathrm{L}$, et al. p50 nuclear factor-kappaB overexpression in tumorassociated macrophages inhibits M1 inflammatory responses and antitumor resistance. Cancer Res. 2006;66:11432-40.

46. Guiducci C, Vicari AP, Sangaletti S, Trinchieri G, Colombo MP. Redirecting in vivo elicited tumor infiltrating macrophages and dendritic cells towards tumor rejection. Cancer Res. 2005;65: 3437-46. 